\title{
Reduced core transport in T-10 and TEXTOR discharges at rational surfaces with low magnetic shear
}

\author{
K.A. Razumova ${ }^{1}$, A.J.H. Donné ${ }^{2}$, V.F. Andreev ${ }^{1}$, \\ G.M.D. Hogeweij ${ }^{2}$, I.S. Bel'bas ${ }^{1}$, A.A. Borschegovskij ${ }^{1}$, \\ A.Yu. Dnestrovskij ${ }^{1}$, V.V. Chistyakov ${ }^{1}$, R. Jaspers ${ }^{2}$, A.Ya. Kislov ${ }^{1}$, \\ V.I. I'lin ${ }^{1}$, D.A. Krupin ${ }^{1}$, S.V. Krylov ${ }^{1}$, D.E. Kravtsov ${ }^{1}$, Y. Liang ${ }^{3}$, \\ S.E. Lysenko ${ }^{1}$, M.V. Maslov ${ }^{1}$, E. Min ${ }^{2}$, T.B. Myalton ${ }^{1}$, \\ G.E. Notkin ${ }^{1}$, M.V. Ossipenko ${ }^{1}$, V.V. Piterskij ${ }^{1}$, D.P. Petrov ${ }^{1}$, \\ I.N. Roi ${ }^{1}$, D.V. Ryzhakov ${ }^{1}$, D.A. Shelukhin ${ }^{1}$, A.V. Sushkov ${ }^{1}$, \\ V.M. Trukhin ${ }^{1}$, V.A. Vershkov ${ }^{1}$, E. Westerhof ${ }^{2}$, T-10 team $^{1}$ and \\ TEXTOR team ${ }^{3}$

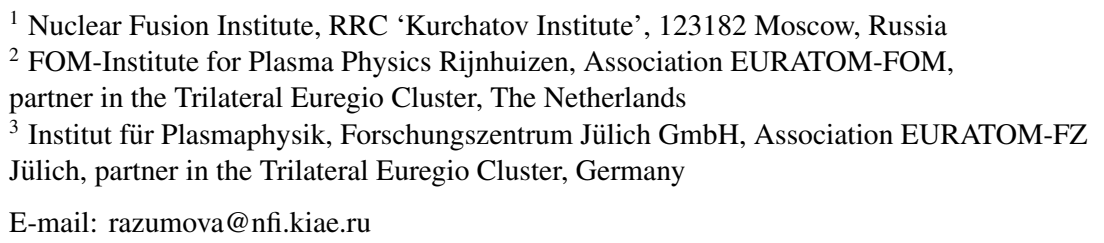

Received 2 March 2004, accepted for publication 3 August 2004

Published 9 September 2004

Online at stacks.iop.org/NF/44/1067

doi:10.1088/0029-5515/44/10/002

\begin{abstract}
It has been observed in the T-10 tokamak that immediately after off-axis electron cyclotron resonance heating (ECRH) switch-off, the core electron temperature stays constant for some time, which can be as long as several tens of milliseconds, i.e. several energy confinement times $\left(\tau_{E}\right)$, before it starts to decrease. Whether or not the effect is observed depends critically on the local magnetic shear in the vicinity of the $q=1$ rational surface, which should be close to zero. It is hypothesized that a small shear can induce the formation of an internal transport barrier. Measurements of density fluctuations in the transport barrier with a correlation reflectometer show immediately after the ECRH switch-off a clear reduction in the fluctuation level, corroborating the above results. The delayed temperature decrease has also been observed in similar discharges in the TEXTOR tokamak; however, the delay is restricted to $\sim 1 \times \tau_{E}$.
\end{abstract}

PACS numbers: 52.55.Fa, 52.50.Sw, 52.25.Fi, 52.35.Ra

(Some figures in this article are in colour only in the electronic version)

\section{Introduction}

The importance of confinement scenarios featuring internal transport barriers (ITBs) for future generation fusion devices, like ITER, is obvious. Whether or not the presence of ITBs in ITER is desirable, there is general agreement that they need to be actively controlled. Based on present knowledge one would like to draw conclusions on the possibilities for ITB control on ITER. But attempts to extrapolate the ITB characteristics as observed on present tokamaks to ITER parameters are not promising. For example, scalings for the power threshold that is needed for the ITB formation differ largely for different authors [1]. This is not surprising since the scaling approach uses the values of local parameters to some power, while the actual processes depend on their profiles (gradients). To be able to make a more accurate extrapolation, it is important to understand the physics of the underlying processes and to find out the necessary conditions for ITB formation. This calls for well-interpreted experiments. As will be shown in the following, discharges with electron cyclotron resonance heating and current drive (ECRH and ECCD) can be used very effectively for this. 

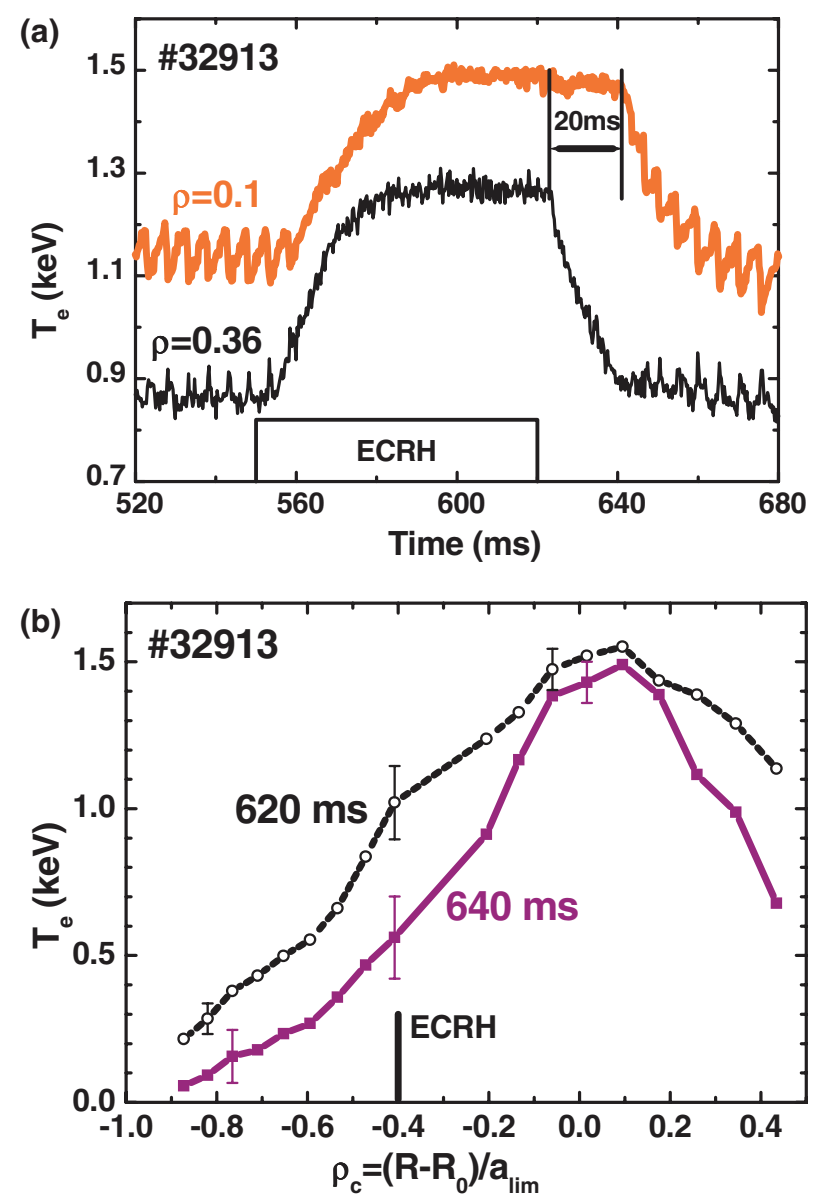

Figure 1. (a) Delayed decrease in the core electron temperature in T-10 after ECRH switch-off at $620 \mathrm{~ms} ; B_{\mathrm{t}}=2.33 \mathrm{~T}, I_{\mathrm{p}}=180 \mathrm{kA}$, $\bar{n}_{\mathrm{e}}=1.5 \times 10^{19} \mathrm{~m}^{-3}, P_{\mathrm{ECRH}}=400 \mathrm{~kW}, q_{\mathrm{lim}}=3.98 ;(b) T_{\mathrm{e}}$ profiles before ECRH switch-off and $20 \mathrm{~ms}$ after; $\rho_{\mathrm{c}}$ is the dimensionless minor radius with respect to the centre of the chamber.

Shortly after ITBs were discovered in JT-60U [2], the existence of multiple ITB regions (sometimes very narrow) were found in RTP experiments in the vicinity of low order rational $q$-surfaces [3]. More recently T-10 experiments with ECCD [4] have led to the following criterion:

The necessary condition for the appearance of zones with

reduced transport is a low value of $\mathrm{d} q / \mathrm{d} \rho$ in the vicinity

of rational $q$-surfaces with low $m$ - and $n$-numbers.

In subsequent experiments at $\mathrm{T}-10$ it was shown that even a small decrease in $\mathrm{d} q / \mathrm{d} \rho$ near the rational magnetic surface leads to an obvious ITB formation ( $\rho$ is the dimensionless flux radius, defined with respect to the magnetic axis of the plasma). A very interesting phenomenon connected to the above findings has been observed in some T-10 regimes with off-axis ECRH [5]. After the off-axis ECRH switch-off, the core electron temperature, $T_{\mathrm{e}}$, does not decrease immediately and continuously in time towards the Ohmic value, as can be expected for a heat-diffusivity coefficient, $\chi_{\mathrm{e}}$, that is constant in time over the plasma column. Instead, the core $T_{\mathrm{e}}$ first stays constant for several tens of milliseconds (figure 1) before it starts to decrease. Sometimes even a slight increase in core energy content is observed. The fact that the central electron temperature remains constant for some time after ECRH switch-off, while the off-axis temperature decreases at the same time (and hence $\nabla T_{\mathrm{e}}$ increases) under the action of constant internal (only $\mathrm{OH}$ ) power deposition, leads to the conclusion that the transport coefficients must be reduced.

The aim of this paper is to investigate the phenomenon of delayed $T_{\mathrm{e}}$ decrease after the off-axis ECRH switch-off and to study whether there is a connection between the heat transport coefficients and the local value of $\mathrm{d} q / \mathrm{d} \rho$. This research is done at the T-10 and TEXTOR tokamaks, under rather similar discharge conditions.

\section{Experimental set-up}

Detailed experiments were performed in the T-10 tokamak ( $R_{0}=1.5 \mathrm{~m}, a_{\mathrm{lim}}=0.3 \mathrm{~m}$, circular plasma cross section). Off-axis ECRH was used to suppress the sawtooth activity in the plasma core. The basic plasma scenario used in this study had a plasma current $I_{\mathrm{p}}=180 \mathrm{kA}$ and a toroidal magnetic field $B_{\mathrm{t}}=2.33 \mathrm{~T}$. The total power of four ECRH gyrotrons $(f=140 \mathrm{GHz})$ was varied up to $900 \mathrm{~kW}$. The ECRH power deposition radius, $\rho_{\mathrm{ECRH}}$, was varied by changing $B_{\mathrm{t}}$. The value of the magnetic field corresponding to on-axis heating at $140 \mathrm{GHz}$ is $B_{\mathrm{t}}=2.5 \mathrm{~T}$. It was observed that the power of only two gyrotrons $\left(P_{\mathrm{ECRH}}=400 \mathrm{~kW}\right)$ is already sufficient for sawtooth stabilization in the given plasma scenario, provided that $\rho_{\mathrm{ECRH}}$ is located slightly outside the sawtooth inversion radius, $\rho_{\mathrm{s}}$.

The electron density profile, $n_{\mathrm{e}}(\rho)$, was measured using an eight-channel microwave interferometer as well as using an eight-channel $\mathrm{HCN}$-laser interferometer, so that the distance between the channels was $4 \mathrm{~cm}$ and the temporal resolution was about $1 \mathrm{~ms}$. The electron temperature profile, $T_{\mathrm{e}}(\rho)$, was measured using a 25-channel electron cyclotron emission (ECE) diagnostic, albeit for the used values of $B_{\mathrm{t}}$ a number of channels were located at the plasma periphery, whereas a few others were located too close to the ECR position, resulting in too high a noise level. So, effectively only 20 ECE channels could be used. The distance between the ECE channels was $2-3 \mathrm{~cm}$, with a gap near the ECR deposition region. The ECE measurements have a temporal resolution of about $15 \mu \mathrm{s}$. The temperature profile, $T_{\mathrm{e}}(\rho)$, was also deduced from the slope of the soft X-ray (SXR) spectra by pulse height analysis (PHA) with a time resolution of $50 \mathrm{~ms}$. The radial position of PHA was changed from shot to shot. The PHA measurements were also used to cross-calibrate the ECE diagnostic. From the absolute intensity of the PHA spectra, together with $T_{\mathrm{e}}$ determined from the SXR continuum slope and the density, $n_{\mathrm{e}}(\rho)$, measured by the interferometers, the $Z_{\text {eff }}$ value for the central part of the plasma could be retrieved. In doing this, it was assumed that carbon is the main plasma impurity. In other experiments it was observed that the contributions of iron-like impurities do not determine the SXR continuum intensity. Because of the exponential dependence of the SXR intensity on $T_{\mathrm{e}}$, the emitted region was restricted to $\rho \leqslant 0.24$ for the plasmas under consideration. The value of $Z_{\text {eff }}$ was also derived from the absolute intensity of the Bremsstrahlung in the visible region. In shots with sawtooth oscillations a rude estimation of $Z_{\text {eff }}$ for $\rho \leqslant \rho_{\mathrm{s}}$ and for a given $B_{\mathrm{t}}$, could also be derived from $T_{\mathrm{e}}(0)$, 
the loop voltage, $V_{1}$, and the $\rho_{\mathrm{s}}$ values $\left(\rho_{\mathrm{s}}=0.2\right.$ in the Ohmic phase of shot \#32913).

The density fluctuation spectra in T-10 were measured as a function of time and space using a heterodyne O-mode correlation reflectometer [6]. Reflectometry can access the region near the axis because the density profile inside the heating zone has a pronounced bell-like shape. This profile is formed during the ECRH heating phase and persists after the heating is switched off. A radial scan of the reflection layer was obtained from shot to shot by a small variation of the discharge density within a $\pm 7 \%$ range while keeping the reflectometer frequency constant. Such a small density variation did not influence the physical processes occurring after ECRH switchoff. The signals received by two antennae, poloidally separated by an angle of $5.8^{\circ}$, were analysed. The value of the phase fluctuations and the integral coherency of the two signals were processed as described in [6].

Experiments have also been done in the TEXTOR tokamak $\left(R_{0}=1.75 \mathrm{~m}, a_{\mathrm{lim}}=0.445 \mathrm{~m}\right.$, circular plasma cross section) in which either $300 \mathrm{~kW}$ of $110 \mathrm{GHz}$ ECRH (maximum pulse length $0.2 \mathrm{~s}$ ) was deposited at the low-field side (LFS) or up to $800 \mathrm{~kW}$ of $140 \mathrm{GHz}$ ECRH $(\leqslant 3 \mathrm{~s})$ at the high-field side (HFS). Changing the magnetic field, $B_{\mathrm{t}}$, on a shot-to-shot basis was used to vary the ECRH power deposition radius. The response of the plasma temperature was measured using a number of heterodyne ECE systems [7] and SXR cameras ( 80 channels), each at a time resolution of $100 \mu \mathrm{s}$. As in T-10, the value of $Z_{\text {eff }}$ was derived from the absolute intensity of the Bremsstrahlung in the visible region, and the $Z_{\text {eff }}$ profile could be deduced from the value of the loop voltage, $V_{1}$, and that of $\rho_{\mathrm{s}}$.

The experimental results of both T-10 and TEXTOR were analysed using the ASTRA transport code [8]. The COBRA code $[9,10]$ was used to calculate the ECRH power deposition profile and to model the dynamics of the transport coefficient profiles at the end of the heating pulse. In all model calculations the experimental parameter profiles were used as input.

\section{Experimental results}

\section{1. $T-10$}

Figure 2 shows the heat diffusivity calculations for the experiment presented in figure 1 . The heat diffusivity, $\chi_{\mathrm{e}}$, calculated by ASTRA for the $\mathrm{OH}$ case and off-axis ECRH phase are shown by dashed and solid lines, respectively. The dash-dotted line corresponds to the $\chi_{\mathrm{e}}$ calculated by COBRA for the dynamic part of the process $(\Delta t=20 \mathrm{~ms})$ after the off-axis ECRH switch-off. The value of $\chi_{\mathrm{e}}$ reduces at all radii (compared with ECRH), and in the core it reaches the very low value of $0.1-0.2 \mathrm{~m}^{2} \mathrm{~s}^{-1}$. Analysis of the influence of the accuracy of $T_{\mathrm{e}}$ measurements on the determination of $\chi_{\mathrm{e}}$ was carried out in [10]. It was shown that the $10 \%$ stochastic errors in $T_{\mathrm{e}}$ lead to $15 \%$ errors in $\chi_{\mathrm{e}}$.

Estimations of $j(\rho)$ and $q(\rho)$ by ASTRA [9] for the $\mathrm{OH}$, the end of the ECRH phase and during the existence of the constant temperature after ECRH switch-off are presented in figure 3. The calculations have been performed with $T_{\mathrm{e}}(\rho)$ and $Z_{\text {eff }}$ taken from the experiment and by using the neoclassical resistance. For the $\mathrm{OH}$ stage, where sawteeth

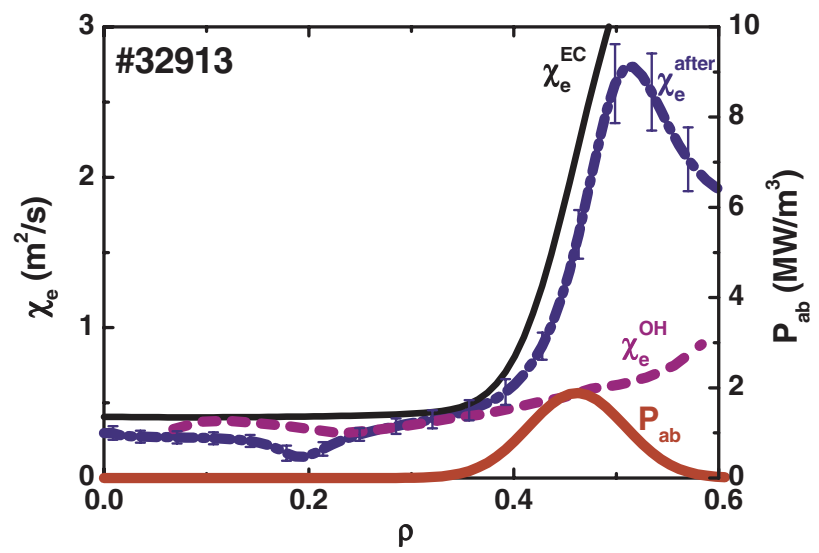

Figure 2. Electron heat diffusivity in the $\mathrm{OH}$ phase (- - - ), at the end of the ECRH phase (-) and during the $T_{\mathrm{e}}$ decay phase $(-\cdot-)$. The COBRA retrieved ECRH power deposition profile, $P_{\mathrm{ab}}$, is also shown.

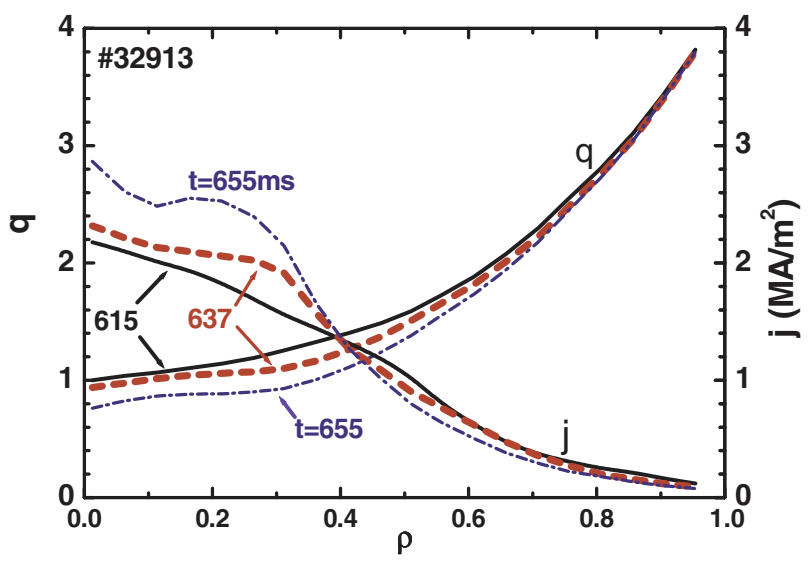

Figure 3. The current density and $q$-profiles during the ECRH phase and after ECRH switch-off in T-10. One can clearly see that $\mathrm{d} q / \mathrm{d} \rho \approx 0$ near $q=1$.

exist, possible changes in the current density profile due to sawtooth crashes have been neglected. The results are consistent with criterion (1) mentioned earlier. The local confinement enhancement leads to a $\nabla T_{\mathrm{e}}$ increase and to a subsequent current density redistribution. This causes in its turn a $\mathrm{d} q / \mathrm{d} \rho$ increase, which then counteracts the $\chi_{\mathrm{e}}$ reduction and the $\nabla T_{\mathrm{e}}$ growth in the barrier region. In other words, a kind of self-consistent relation between $\nabla T_{\mathrm{e}}$ (level of transport) and $\mathrm{d} q / \mathrm{d} \rho$ is established. So, a pure inductive current generation with a monotonic $q(\rho)$ profile always leads to $\mathrm{d} q / \mathrm{d} \rho>0$ in the 'barrier' region, which is not optimal for achieving good confinement. Off-axis ECRH switch-off leads to current density redistribution in the heating region and transiently $\mathrm{d} q / \mathrm{d} \rho \approx 0$, causing a local confinement improvement in this region. One can see that $\mathrm{d} q / \mathrm{d} \rho$ is positive at all radii before the ECRH switch-off, and forms a plateau after it. Later $q(\rho)$ becomes $<1$ and sawtooth relaxations appear, as is also seen in experiment.

In the experiment presented in figure 1 two gyrotrons were used. The power $(400 \mathrm{~kW})$ turned out to be just enough for sawtooth stabilization. However, when instead of two gyrotrons $\left(P_{\mathrm{ab}}=400 \mathrm{~kW}\right)$ four gyrotrons were used $\left(P_{\mathrm{ab}}=900 \mathrm{~kW}\right)$, the persistency of the $T_{\mathrm{e}}(0)$ decrease was not 


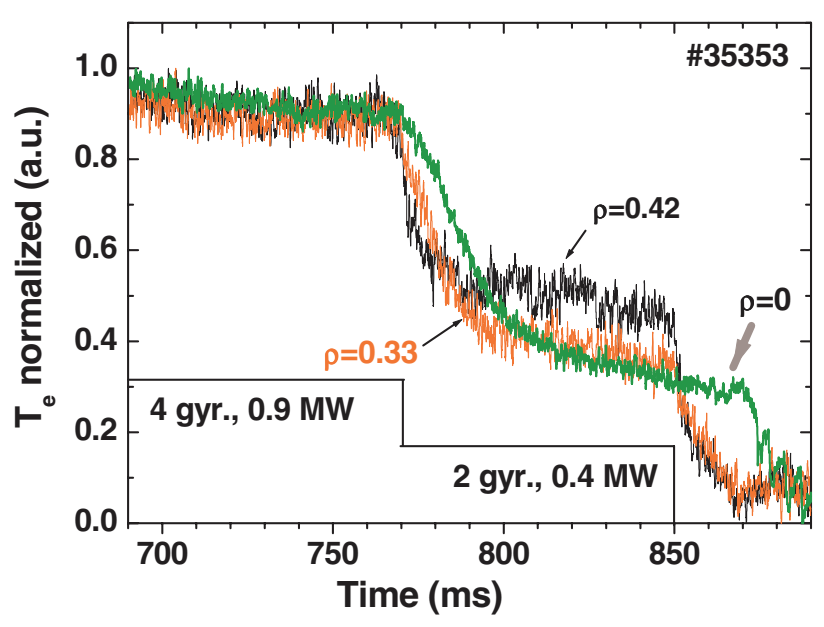

Figure 4. Shot with high ECRH power, which is switched off in two steps. A delayed decrease in $T_{\mathrm{e}}$ is seen only after the second switch-off (thick arrow).

observed after switch-off of all gyrotrons $(900 \mathrm{~kW})$. The effect was also absent when two of the four gyrotrons were switched off. However, the delayed $T_{\mathrm{e}}(0)$ decrease was observed when the two remaining gyrotrons were switched off (figure 4). As sawteeth were suppressed in both cases, the difference was at the level of ECRH power only. When two gyrotrons are used, we obtain $q_{\text {core }} \approx 1$; in the case of four gyrotrons $q_{\text {core }}>1$.

To investigate whether $q_{\text {core }} \approx 1$ is an important condition for the observed confinement improvements, the following experiments were done:

(1) When two of the four gyrotrons are operated for a short time only, then both $j(\rho)$ redistribution and $q_{\text {core }}$ increase do not take place, and a delayed $T_{\mathrm{e}}(0)$ decrease is observed. Figure 5(a) shows that after two of the four gyrotrons were switched off ( $40 \mathrm{~ms}$ after the off-axis ECRH switch-on), $T_{\mathrm{e}}(0)$ at least remains constant (perhaps, it even continues to increase). A delay in the $T_{\mathrm{e}}(0)$ decrease of $t_{\mathrm{del}}=9 \mathrm{~ms}$ was observed.

(2) When the four gyrotrons were used at a higher value of the plasma current $I_{\mathrm{p}}$, such that again the sawteeth were just stabilized and $q_{\text {core }}$ was near unity, a delayed $T_{\mathrm{e}}(0)$ decrease was also seen after simultaneous switch-off of all gyrotrons. Figure 5(b) shows the regime with higher current: $B_{\mathrm{t}}=2.3 \mathrm{~T}$; $I_{\mathrm{p}}=225 \mathrm{kA} ; \bar{n}_{\mathrm{e}}=1.8 \times 10^{19} \mathrm{~m}^{-3}$. In this case the four gyrotrons just stabilized the sawtooth activity. The value of $B_{\mathrm{t}}$ was adapted such that the ECRH power is deposited just outside the sawtooth inversion radius, $\rho_{\mathrm{s}}$, which increases for higher current. After switching off the four gyrotrons the value of $T_{\mathrm{e}}(0)$ slowly decreases - and even stays constant for a short time, before it strongly decreases after a delay of $28 \mathrm{~ms}$.

(3) One can also observe a delayed $T_{\mathrm{e}}(0)$ decrease after switch-off of the four gyrotrons at low plasma current $I_{\mathrm{p}}=$ $180 \mathrm{kA}$. This is possible if one uses preliminary on-axis heating (with up to $450 \mathrm{~kW}$ at $130 \mathrm{GHz}$ ). The on-axis heating makes it possible to obtain the desirable $q$-profile when applying high power off-axis heating with four gyrotrons. It turned out that even a quarter of the on-axis gyrotron power $\left(P_{\mathrm{ab}} \leqslant 120 \mathrm{~kW}\right)$ is too much for achieving sawtooth stabilization with the four off-axis $140 \mathrm{GHz}$ gyrotrons. Nevertheless, again a delayed $T_{\mathrm{e}}(0)$ decrease was seen with a delay of $15 \mathrm{~ms}$, even though
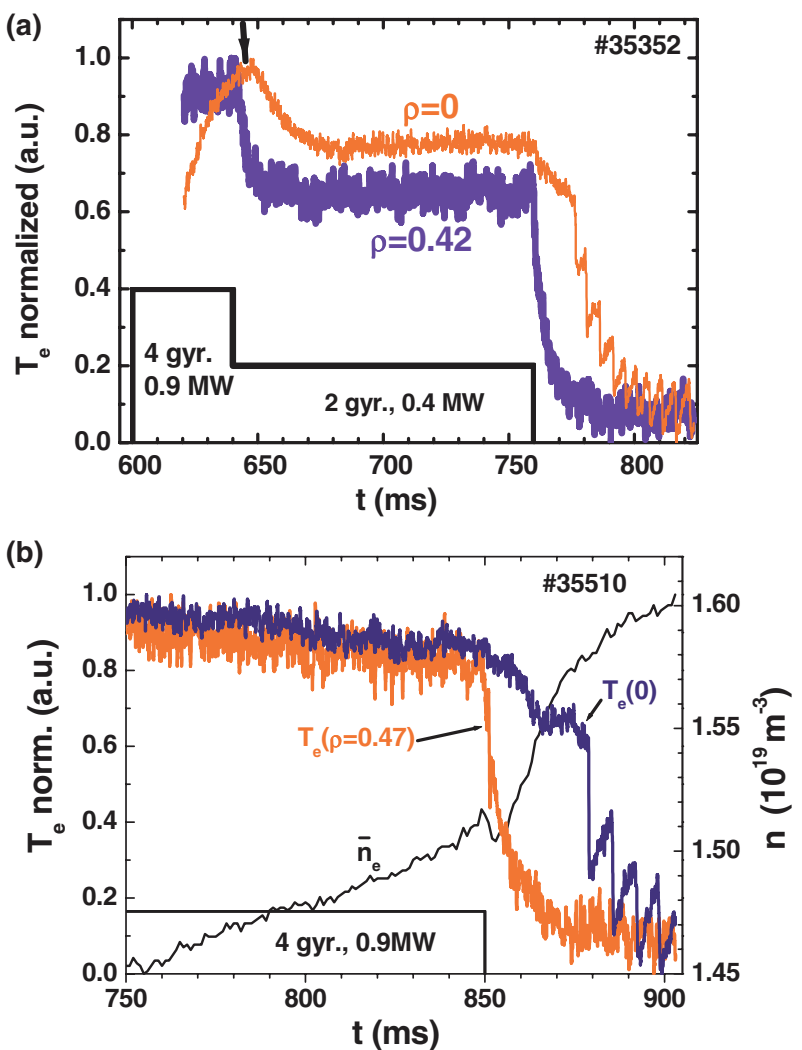

Figure 5. (a) Shot where two of the four gyrotrons are operated only for a short time $(40 \mathrm{~ms}) . T_{\mathrm{e}}(0)$ continues to grow after ECRH switch-off (thick arrow); $(b)$ shot with higher current $\left(B_{\mathrm{t}}=2.3 \mathrm{~T}\right.$; $\left.I_{\mathrm{p}}=225 \mathrm{kA} ; \bar{n}_{\mathrm{e}}=1.8 \times 10^{19} \mathrm{~m}^{-3}, q_{\text {lim }}=3.2\right)$. A delayed $T_{\mathrm{e}}(0)$ decrease is observed after simultaneous switch-off of all four gyrotrons.

the sawteeth were not completely suppressed (figure 6(a)). This indicates that the stabilization of sawtooth activity is not a necessary condition for the delayed $T_{\mathrm{e}}(0)$ decrease. In contrast, it is important to have a $q(\rho)$ profile with a low shear $\mathrm{d} q / \mathrm{d} \rho$ in the vicinity of $q=1$.

Let us finally compare two T-10 shots with the same initial plasma parameters $\left(B_{\mathrm{t}}=2.33 \mathrm{~T}, I_{\mathrm{p}}=185 \mathrm{kA}, \bar{n}_{\mathrm{e}}=1.4 \times\right.$ $10^{19} \mathrm{~m}^{-3}$ ), but with different off-axis ECRH input powers: shot \#35355 with two gyrotrons and shot \#35358 with four gyrotrons (figure $7(a)$ ). In the case of two gyrotrons, $T_{\mathrm{e}}(0)$ remains constant for $25 \mathrm{~ms}$ after switch-off. In the case of four gyrotrons, $T_{\mathrm{e}}(0)$ immediately decreases after switch-off until it reaches the same value as in the case of off-axis heating with two gyrotrons. Then it remains constant for $15 \mathrm{~ms}$. As can be seen in figure $7(d)$ not only the value of $T_{\mathrm{e}}(0)$, but also the $T_{\mathrm{e}}(\rho)$ profiles are the same in both cases during the period of constant $T_{\mathrm{e}}(0)$. This indicates that the core confinement improves only after the surplus of stored energy the plasma core is lost.

The experimental results strongly point towards the conclusion that a low value of $\mathrm{d} q / \mathrm{d} \rho$ leads to a confinement enhancement only if the local value of $q$ is near a rational value (in the present case when $q$ is near unity). It is important to estimate the range of $q=q_{\mathrm{r}} \pm \delta q$ around the rational value $q_{\mathrm{r}}$ where the ITB exists.

The analysis of the delayed $T_{\mathrm{e}}(0)$ decrease after ECRH switch-off was carried out under the assumption that 

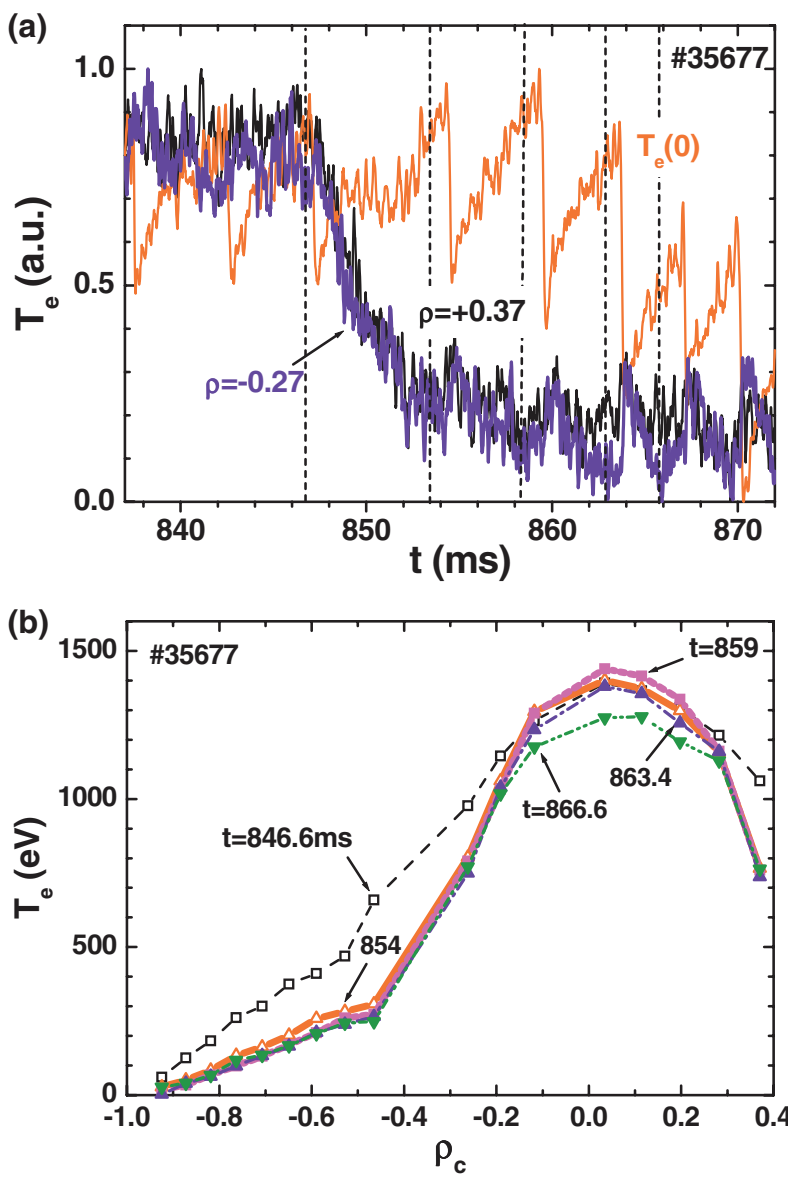

(c)

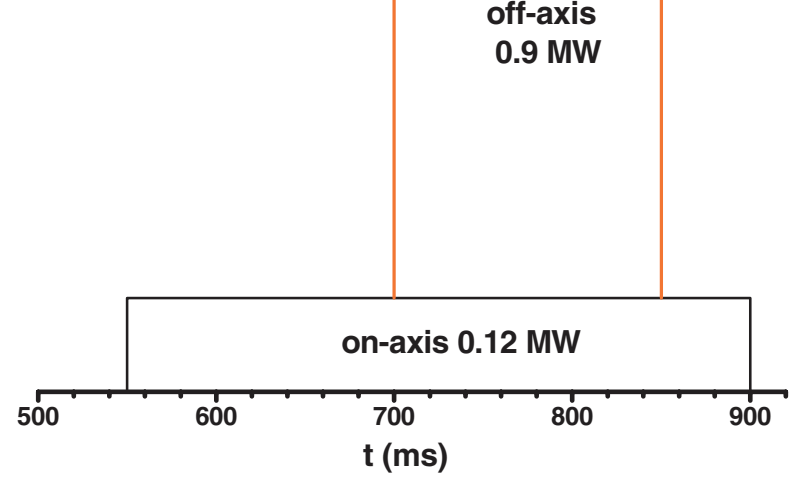

Figure 6. On-axis ECRH at $130 \mathrm{GHz}(120 \mathrm{~kW})$ combined with off-axis ECRH at $140 \mathrm{GHz}(900 \mathrm{~kW})$ using four gyrotrons in T-10. In spite of the sawtooth activity, the resulting $q$-profile at $\rho \geqslant \rho_{\mathrm{s}}$ is similar to that in the experiment of figure 1 . A delayed $T_{\mathrm{e}}(0)$ decrease is seen after off-axis ECRH switch-off: $(a)$ the normalized ECE intensity proportional to $T_{\mathrm{e}} ;(b) T_{\mathrm{e}}(\rho)$ profiles before and after off-axis ECRH switch-off for the time slices indicated in $(a) ;(c)$ the scenario of ECRH.

the electron ITB is formed in the region of the rational surface $q_{\mathrm{r}}=1$. The $q$-profile was calculated by the ASTRA code using the experimental temperature profiles. It was concluded that $\delta q \approx 0.25$ and, hence, the barrier forms in the range $1<$ $q<1.25$. The results of calculations for T-10 shots \#35355 and \#35358 are shown in figures 7(b) and $(c)$, where $\mathrm{d} q / \mathrm{d} \rho$ is the dimensionless gradient of $q$ at the radius of $q=1.25$.
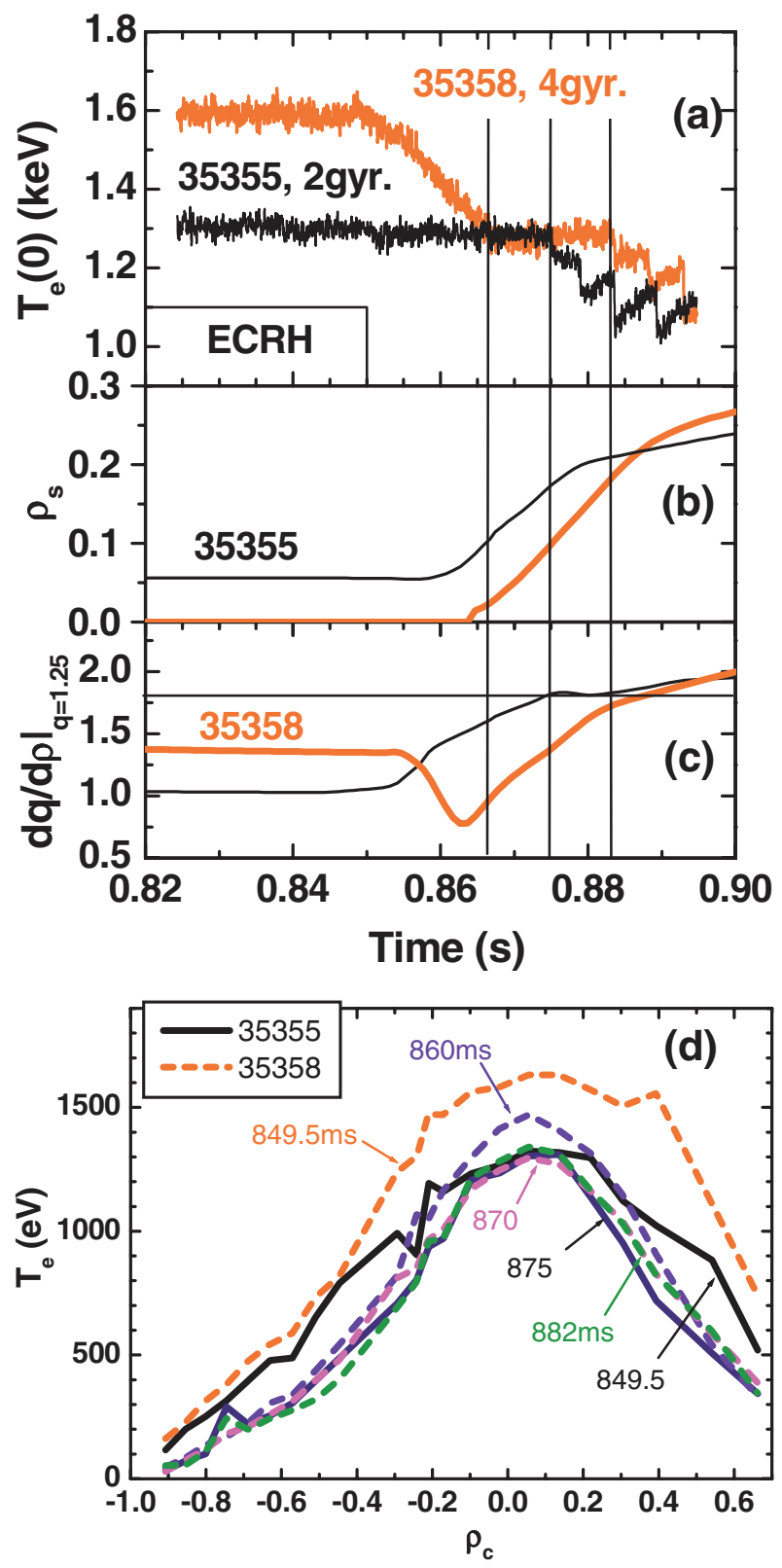

Figure 7. (a) Evolution of the plasma parameters after ECRH switch-off for T-10 shots \#35355 and \#35358. At high heating power (shot \#35358) the core temperature decays until it reaches the same level as in a similar discharge with a lower heating power (shot \#35355). After this moment the temperature decrease temporarily stops; $(b)$ position of the $q=1$ surface calculated using ASTRA; $(c)$ calculations of the value of $\mathrm{d} q / \mathrm{d} \rho$ at $q=1.25$;

(d) temperature profiles after ECRH switch-off in these shots.

As can be seen from figure $7(a)$, the $T_{\mathrm{e}}(0)$ value for shot \#35355 starts to decrease with a $25 \mathrm{~ms}$ delay after the ECRH switch-off. The $T_{\mathrm{e}}(0)$ decrease starts when the value of $\mathrm{d} q / \mathrm{d} \rho$ exceeds 1.6. At this moment the ITB vanishes. In shot \#35358 the $T_{\mathrm{e}}(0)$ value immediately starts to decrease after ECRH switch-off until it reaches $865 \mathrm{~ms}$ the same level as that in shot \#35355. In spite of a very low $\mathrm{d} q / \mathrm{d} \rho$ at $t=860 \mathrm{~ms}$, confinement improvement is not observed because the $q_{\mathrm{r}}=1$ surface does not exist. At $t=865 \mathrm{~ms}$ the resonant surface $q_{\mathrm{r}}=1$ appears in the plasma (figure $7(b)$ ), the ITB is formed 


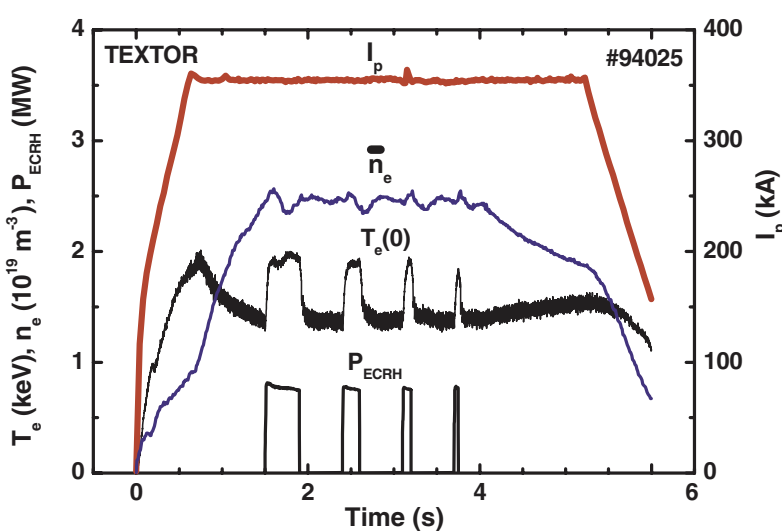

Figure 8. Scenario of ECRH switch-off experiment in TEXTOR.

and continues to exist until $t=883 \mathrm{~ms}$. At that time $\mathrm{d} q / \mathrm{d} \rho$ reaches approximately the same critical value of 1.6 as for \#35355. At that moment the ITB disappears and the $T_{\mathrm{e}}(0)$ value starts to decrease. We can conclude from our calculations that the presence of the $q_{\mathrm{r}}=1$ resonant surface and the low value of $\mathrm{d} q / \mathrm{d} \rho$ in the vicinity of this surface are the necessary conditions for the existence of an ITB. In the T-10 experiment we find

$$
\left.\frac{\mathrm{d} q}{\mathrm{~d} \rho}\right|_{q=1.25}<1.6 .
$$

One can conclude that the value of $\mathrm{d} q / \mathrm{d} \rho$ near a rational surface can be considered as the control parameter for the ITB formation. The calculated critical value of $\mathrm{d} q / \mathrm{d} \rho$ is only an estimate. To determine this value more precisely measurements of the $q$-profile are needed.

\subsection{TEXTOR}

In TEXTOR, ECRH experiments were performed with different positions of the EC resonance $\rho_{\mathrm{ECRH}}$. Sawteeth were stabilized for $\rho_{\mathrm{ECRH}}$ outside the $q=1$ radius both for LFS heating at $110 \mathrm{GHz}$ [11] and HFS heating at $140 \mathrm{GHz}$. When this stabilization just takes place ( $\rho_{\mathrm{ECRH}}$ just outside $q=1$ ) the same phenomenon as in T-10 is observed, most clearly for HFS heating. In this paper we will concentrate on the results at $140 \mathrm{GHz}$ deposited at the HFS, where the effect is most pronounced.

In a series of discharges with HFS ECRH with $\rho_{\mathrm{ECRH}}$ near or just outside the $q=1$ radius $\left(B_{\mathrm{t}}=2.32-2.36 \mathrm{~T}\right)$, four pulses of ECRH were applied, with a length of $400 \mathrm{~ms}$, $200 \mathrm{~ms}, 100 \mathrm{~ms}$ and $50 \mathrm{~ms}$, respectively (figure 8). The Ohmic phase between the pulses had a length of $500 \mathrm{~ms}$, i.e. a few current diffusion times, to allow the current density profile to relax to its Ohmic state. Interestingly, the longest delay of the central $T_{\mathrm{e}}$ decay $\left(\sim 20 \mathrm{~ms}\right.$, i.e. $\left.\sim 1 \times \tau_{E}\right)$ was observed after switch-off of the shortest ECRH pulse (figure 9). ECE $T_{\mathrm{e}}$ and SXR emissivity time traces are presented in figures 9 and 10 , respectively. Figure 11 shows the ECE $T_{\mathrm{e}}$ profiles. As in the T-10 case, the heat is conserved inside the $q=1$ region. Figure 12 shows the results of analysis using the COBRA and ASTRA codes. The value of $\chi_{\mathrm{e}}$ is strongly reduced in the area outside $\rho_{\mathrm{ECRH}} \sim 0.3$ after ECRH switch-off.

The effect on the TEXTOR plasma with $110 \mathrm{GHz}$ LFS heating [10] is far less pronounced than with $140 \mathrm{GHz}$ HFS

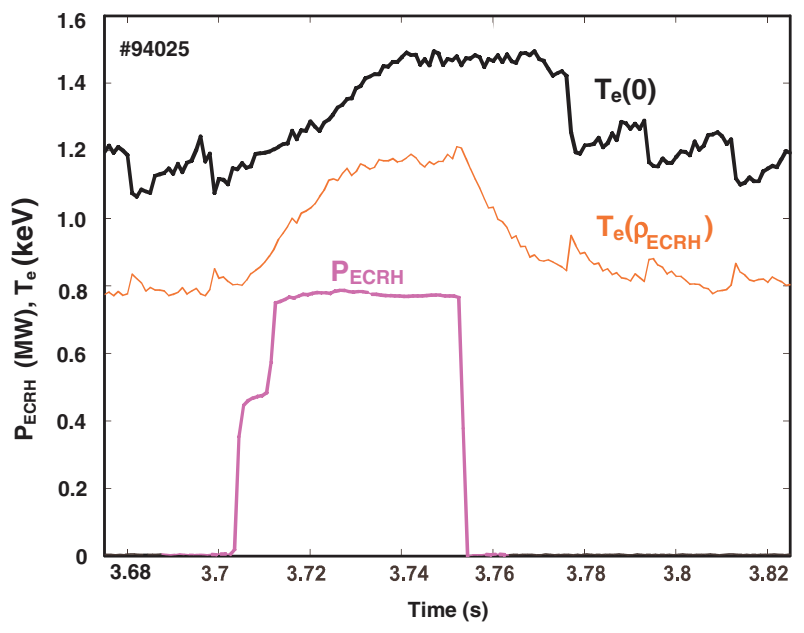

Figure 9. Time evolution of the electron temperature in the vicinity of the short ECRH pulse in TEXTOR discharge \#94025, $q_{\mathrm{lim}}=3.7$. Note the immediate response of $T_{\mathrm{e}}\left(\rho_{\mathrm{ECRH}}\right)$ upon ECRH switch-off and the delayed response (by $\sim 20 \mathrm{~ms}$ ) of $T_{\mathrm{e}}(0)$.

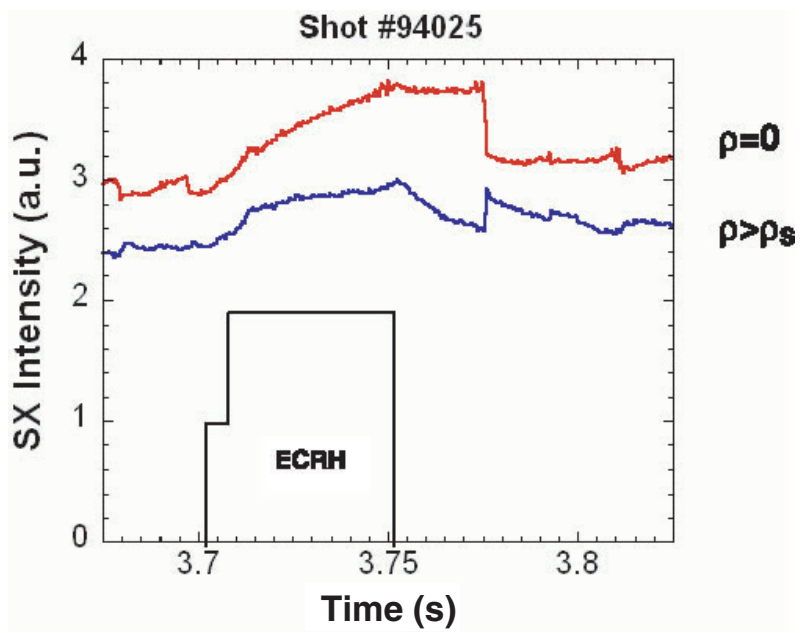

Figure 10. Traces of the SXR emissivity after switch-off of a short ECRH pulse in TEXTOR discharge \#94025 measured through the plasma centre and through an area outside $q=1$.

heating. Most probably this can be attributed to the fact that the ECRH power used on the LFS was much lower; moreover the density of magnetic surfaces is higher on the LFS. The fact that the observed delay in T-10 is much longer than in TEXTOR (in terms of $\tau_{E}$ ) may be due to the fact that the specific ECRH power was lower than in $\mathrm{T}-10$.

\section{Behaviour of turbulence in T-10 during improved confinement}

The density fluctuations in T-10 were measured by means of $\mathrm{O}$-mode reflectometry. It was estimated that for reflections at radii $\rho=0.2-0.5$; the one-dimensional analysis models yield an error $\leqslant 20 \%$ for poloidal wavelengths $>1.5 \mathrm{~cm}$. As the present poloidal correlation reflectometry measurements are for wavelengths greater than $2 \mathrm{~cm}$, the one-dimensional analysis models should be valid.

The radial dependence of the relative amplitude of density fluctuations, $\sqrt{\left\langle\delta n^{2}\right\rangle} / n$, and the spectral coherence 


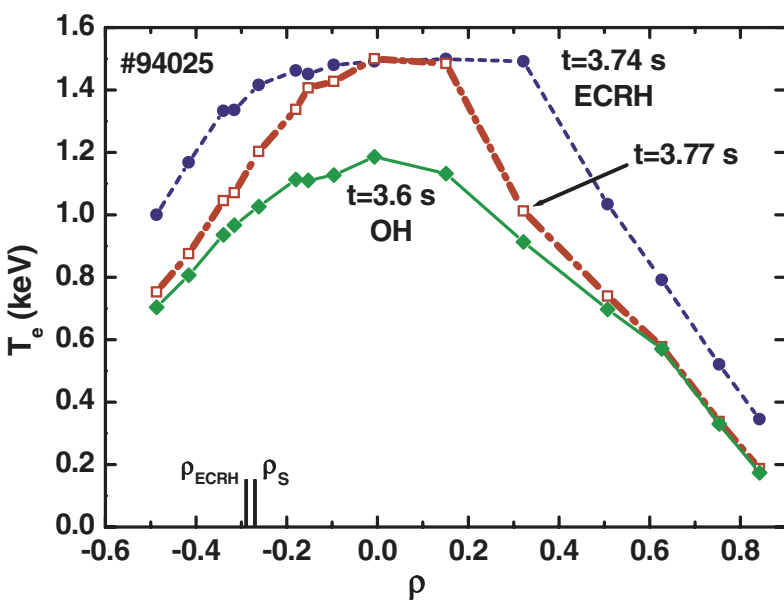

Figure 11. $T_{\mathrm{e}}$ profiles as measured by ECE for the same TEXTOR discharge as in figure 8, in the Ohmic phase (3.6s), during ECRH $(3.74 \mathrm{~s})$ and $18 \mathrm{~ms}$ after switch-off of the $50 \mathrm{~ms}$ ECRH pulse $(3.772 \mathrm{~s})$.

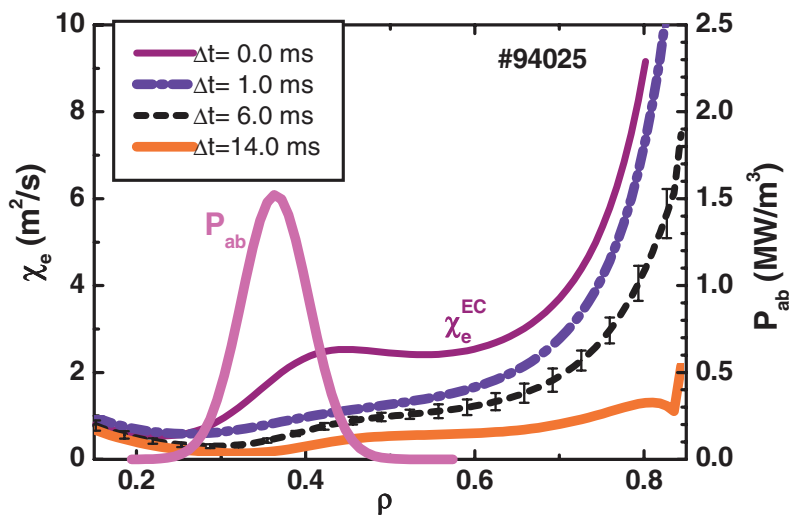

Figure 12. Heat diffusivity, $\chi_{e}$, in TEXTOR calculated using ASTRA before, and using COBRA for various time instants during the delayed $T_{\mathrm{e}}(0)$ decrease after ECRH switch-off at $t=3.77 \mathrm{~s}$. The COBRA retrieved ECRH power deposition profile, $P_{\mathrm{ab}}$, is also shown.

between two poloidally separated channels are presented in figures $13(a)$ and $(b)$, respectively. The quantities were evaluated for the Ohmic phase of the discharge and during the time window of 5-15 ms after ECRH switch-off. A pronounced decrease in the relative density fluctuation level as well as in the spectral coherence are observed in the radial range $0.25<\rho<0.35$. This observation supports the earlier conclusion that an ITB is formed outside the radial position of the $q=1$ surface, $\rho_{\mathrm{s}}$. The decrease in the rms of the relative density fluctuations by a factor of 4 is consistent with the considerable decrease in the electron heat conductivity seen in figures 2 and 11.

\section{Discussion}

The experimental results strongly point towards the conclusion that a low value of $\mathrm{d} q / \mathrm{d} \rho$ leads to a confinement enhancement only if the local value of $q$ is near a rational value (in the present case when $q_{\mathrm{r}}=1$ ).

The fact that the strongest effect in TEXTOR is seen after the shortest ECRH pulses nicely fits into the above
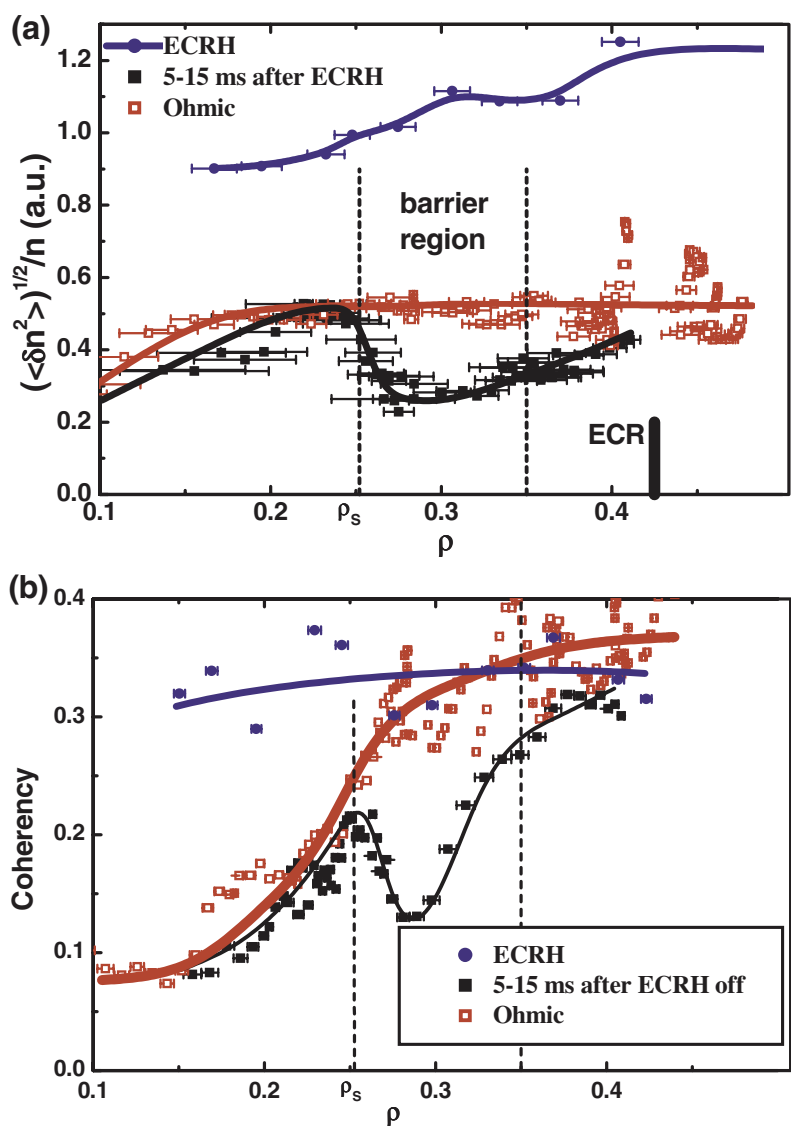

Figure 13. Relative density fluctuation level in the frequency band $35-400 \mathrm{kHz}(a)$ and the spectral coherence $(b)$ measured $5-15 \mathrm{~ms}$ after ECRH switch-off in T-10. The Ohmic values are given for reference. Reduction of level and coherency after ECRH switch-off provides evidence of the transient ITB formation in the region $0.25<\rho<0.35$.

model: the $q$-profile evolves to a favourable shape in $\sim 50 \mathrm{~ms}$, and then (when ECRH is still on) it further evolves to a less favourable shape.

We still do not understand the physical processes at the rational surfaces, which determine the confinement changes. As was seen previously [4], a low value of $\mathrm{d} q / \mathrm{d} \rho$ near the rational surface would cause an increase in the growth of magnetic islands, which would spoil the plasma confinement. However, in this study the opposite is observed. To improve our understanding we need to perform further studies in which the $q$-profile is also measured as a function of time. These experiments are planned later this year in TEXTOR after the new motional Stark effect diagnostic has been commissioned.

In conclusion it can be stated that for electron ITB formation it is important to have as low as possible a derivative, $\mathrm{d} q / \mathrm{d} \rho$, at the rational magnetic surface. This immediately explains why in reversed shear configurations the ITB is easier formed, and why the transport is decreased more substantially. As was mentioned above, for Ohmic current drive and monotonic $q(\rho)$ the value of $\mathrm{d} q / \mathrm{d} \rho$ is always positive in the ITB region due to the current distribution in the steep $\nabla T_{\mathrm{e}}$ zone. On the other hand, if an ITB is formed in a negative shear region, the current redistribution during ITB formation should lead to a wider region with $\mathrm{d} q / \mathrm{d} \rho \approx 0$ and, hence, to a better confinement than one 
with monotonic $q(\rho)$. One could also consider the use of ECCD. It seems that even a small correction of the $q(\rho)$ profile by ECCD near the rational magnetic surface may help us to achieve a better confinement in the ITB region.

\section{Acknowledgments}

We would like to dedicate this paper to Valery Dreval, who was one of the driving forces behind the T-10-TEXTOR collaboration until he was taken away from us by an unfortunate accident.

We are grateful to Yu.D. Pavlov and V.I. Poznyak for assistance in conducting T-10 experiments. This work at T-10 was supported by DAST Minatom RF and Grants RFBR 04-02-17562, NSh-1608.2003.2. The work at TEXTOR was performed under the EURATOM-FOM association agreement with financial support from the 'Nederlandse Organisatie voor Wetenschappelijk Onderzoek (NWO)', the 'Stichting voor Fundamenteel Onderzoek der Materie (FOM)', EURATOM and the Forschungszentrum Jülich GmbH. The collaborative research between T-10 and TEXTOR was further supported by NWO-RFBR Grant 047.009.009 and INTAS Grant 2001-2056.

\section{References}

[1] Connor J.W. et al 2004 Nucl. Fusion 44 R1

[2] Koide Y. et al 1994 Phys. Rev. Lett. 723662

[3] Lopes Cardozo N.J. et al 1997 Plasma Phys. Control. Fusion 39 B303

[4] Razumova K.A. et al 2000 Plasma Phys. Control. Fusion 42973

[5] Razumova K.A. et al 2003 Plasma Phys. Control. Fusion 45 1247

[6] Vershkov V.A. et al 1999 Rev. Sci. Instrum. 701700

[7] Waidmann G. 1997 Rev. Sci. Instrum. 68492

[8] Pereverzev G.V. and Yushmanov P.N. 2002 ASTRA: Automated System for Transport Analysis in a Tokamak Max-Planck Institute Report IPP 5/98

[9] Andreev V.F. et al 2004 Plasma Phys. Control. Fusion 46319

[10] Andreev V.F., Dnestrovskij Yu.N. and Popov A.M. 1993 Nucl. Fusion 33499

[11] Jaspers R. et al 2002 Proc. 29th EPS Conf. on Control. Fusion and Plasma Phys. (Montreux, 2002) vol 26B (Europhys. Conf. Abstr.) P-2.063 